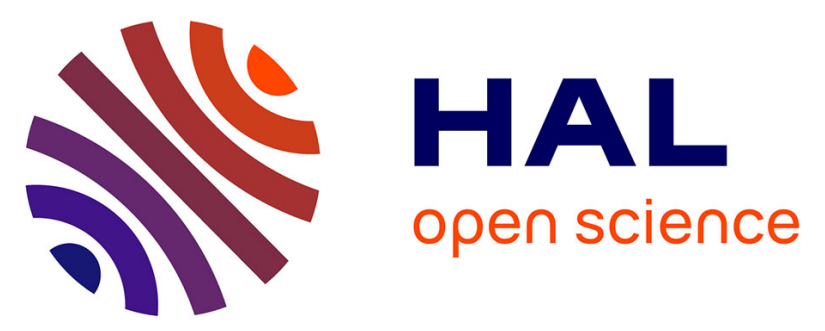

\title{
Investigating Upper Limb Movement Classification on Users with Tetraplegia as a Possible Neuroprosthesis Interface
}

\author{
Lucas Fonseca, Antonio Padilha Lanari Bo, David Guiraud, Benjamin
}

Navarro, Anthony Gélis, Christine Azevedo Coste

\section{To cite this version:}

Lucas Fonseca, Antonio Padilha Lanari Bo, David Guiraud, Benjamin Navarro, Anthony Gélis, et al.. Investigating Upper Limb Movement Classification on Users with Tetraplegia as a Possible Neuroprosthesis Interface. EMBS: Engineering in Medicine and Biology Conference, Jul 2018, Honolulu, United States. pp.5053-5056, 10.1109/EMBC.2018.8513418 . lirmm-01900330

\section{HAL Id: lirmm-01900330 \\ https://hal-lirmm.ccsd.cnrs.fr/lirmm-01900330}

Submitted on 24 Oct 2018

HAL is a multi-disciplinary open access archive for the deposit and dissemination of scientific research documents, whether they are published or not. The documents may come from teaching and research institutions in France or abroad, or from public or private research centers.
L'archive ouverte pluridisciplinaire HAL, est destinée au dépôt et à la diffusion de documents scientifiques de niveau recherche, publiés ou non, émanant des établissements d'enseignement et de recherche français ou étrangers, des laboratoires publics ou privés. 


\title{
Investigating Upper Limb Movement Classification on Users with Tetraplegia as a Possible Neuroprosthesis Interface
}

\author{
Lucas Fonseca ${ }^{1}$, Antônio Bó ${ }^{2}$, David Guiraud ${ }^{3}$, Benjamin Navarro $^{4}$, Anthony Gélis ${ }^{5}$, Christine Azevedo-Coste ${ }^{3}$
}

\begin{abstract}
Spinal cord injury (SCI), stroke and other nervous system conditions can result in partial or total paralysis of individual's limbs. Numerous technologies have been proposed to assist neurorehabilitation or movement restoration, e.g. robotics or neuroprosthesis. However, individuals with tetraplegia often find difficult to pilot these devices. We developed a system based on a single inertial measurement unit located on the upper limb that is able to classify performed movements using principal component analysis. We analyzed three calibration algorithms: unsupervised learning, supervised learning and adaptive learning. Eight participants with tetraplegia (C4C7) piloted three different postures in a robotic hand. We achieved $89 \%$ accuracy using the supervised learning algorithm. Through offline simulation, we found accuracies of $76 \%$ on the unsupervised learning, and $88 \%$ on the adaptive one.
\end{abstract}

\section{INTRODUCTION}

Diseases and lesions of the nervous system can have dramatic consequences for patients. Cases like stroke or spinal cord injury (SCI) can cause partial or complete paralysis of limbs. In the worst cases, individuals lose not only lower limbs motor capabilities, usually relying on a wheelchair for locomotion, but also upper limb motion [7].

Numerous technologies have been proposed as solutions for upper limbs disabilities, such as functional electrical stimulation (FES) [2], and robotic devices [1]. However users with tetraplegia often face challenges on controlling these devices. Relative success has been achieved by either simple interfaces [4] or invasive brain-computer interfaces [12]. Whenever the user still has some controllable upper limb motor capability, it is possible to use that to control a device [6]. The challenge is then to identify the intended movement performed by the user. Electromyography (EMG) has been considered [6], [11], however muscular activitybased control presents some issues like the need for one sensor per muscle. Also, the presence of co-contraction

\footnotetext{
${ }^{1} \mathrm{PhD}$ Candidate at LARA, University of Brasília, Brazil. Invited researcher at INRIA, Université de Montpellier, Montpellier, France. Email: lucasfonseca@lara.unb.br

${ }^{2}$ LARA, University of Brasília, Brazil.

${ }^{3}$ INRIA/Université de Montpellier, Montpellier, France.

${ }^{4}$ Université de Montpellier, LIRMM, Montpellier, France.

${ }^{5}$ PROPARA clinical center, Montpellier, France.

*This work was supported by Coordenação de Aperfeiçoamento de Pessoal de Nível Superior (CAPES), Brazil, and by the LabEx NUMEV incorporated into the I-Site (Montpellier, France).
}

makes it difficult to contract individual muscles and, when FES is present, the stimulation artifacts in the EMG signal have to be considered. Instead of muscle activity, movements can be used as inputs. In [9], postures performed by healthy subjects were classified using a method based on Linear Discriminant Analysis and 2 9-DOF (degrees of freedom) inertial measurement units (IMUs). Additionally, [5] used multiple IMUs and a radial basis function network based regression algorithm to control an elbow prosthesis with residual upper limb movement on amputees. Recently, on [10], three subjects with tetraplegia controlled a powered wheelchair using residual shoulder movement. The method in [10] is based on Principal Component Analysis (PCA) and 4 IMUs.

In this work we propose a system capable of associating shoulder movements to three commands on a robotic hand. It is based on a single IMU with only accelerometer and gyroscope. For that, we developed three classifiers based on PCA. Eight subjects with tetraplegia piloted the robotic hand postures with one classifier, and the other two were later evaluated through simulation.

\section{Subjects And Methods}

Eight patients with tetraplegia were selected at Propara Neurological Rehabilitation Center in Montpellier, France. They had SCI levels C4-C7, American Spinal Injury Association impairment scale A or B and stable medical condition. They were males between 18 and $65(39 \pm 15)$ years old, and had lesion chronocity higher than 6 months ( $14 \pm 17$ years). The protocol was approved by a national ethical committee (CPP 2016-A00711-50) and subjects provided informed consent prior to their participation. They had limited hand movement, but could perform controlled shoulder movement.

For validation purposes we used the Shadow Dexterous Hand (Shadow Robot Company, London, UK), a robotic hand that can reproduce human movements with great fidelity. We set up two different postures on it: in opened position (fingers fully extended); and in closed position (fingers fully flexed) in a grasping attitude. We also configured a rest position (semi-flexed fingers).

One IMU (Hikob@ Fox, Villeurbanne, France) was positioned either on the subject's upper arm or shoulder depending on their ability to execute two distinct movements in a 


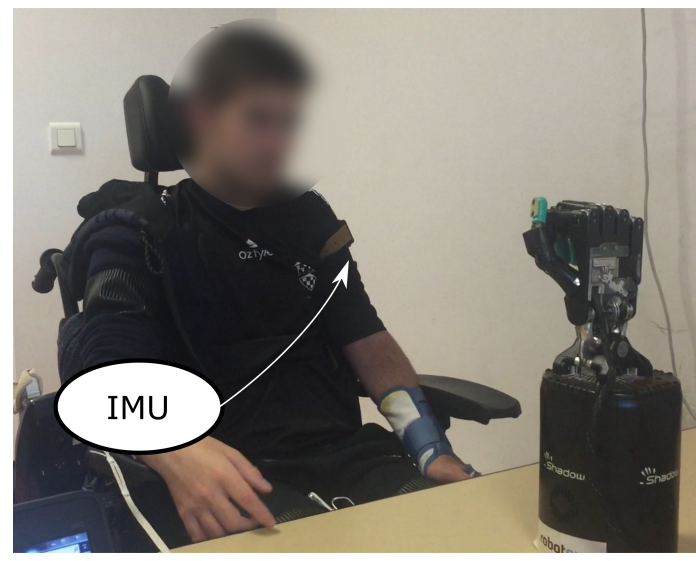

Figure 1. Experimental set-up.

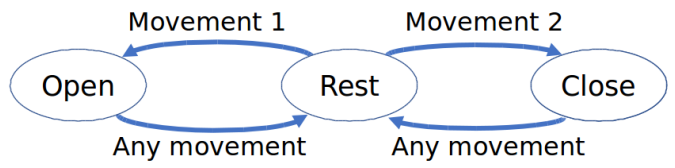

Figure 2. Finite state machine used to control the robotic hand depending on the classified movement performed.

reproducible manner. The IMU communicated wirelessly with a a computer at approximately $50 \mathrm{~Hz}$. It sent 3-axis accelerometer and 3-axis gyroscope data. No magnetometer was used in order to simulate a scenario where low processing power and power consumption are issues, e.g. if it were implanted.

Subjects faced the robotic hand so they could see it react to their movements. The set-up is illustrated on Fig. 1, where the robotic hand is in the closed position.

Two movements were calibrated and associated to two commands to control the robotic hand. Since there are three different positions to be controlled by two movements, the finite state machine depicted on Fig. 2 was employed. Each command had to correctly classify the user's movement to activate the desired posture - robotic hand opened or closed - from the rest position. When in one of these two postures, any movement triggered the rest position.

The experimental protocol consisted of a calibration and a validation phase. On the calibration phase, we asked users to repeatedly perform up to four movements during 10 seconds each. These could be any repeatable and distinct movement, like moving the shoulder up or forward, and returning it to the initial position. On the validation phase we asked them to pilot the robotic hand. Each posture was comprised of two movements: one starting on the rest position, which had to be correctly classified by the system, and one back to the rest position, which had only to be detected, but not classified (see Fig. 2). Each of the two postures (open/close) was executed 5 times in a random order following our instructions.

We developed three algorithms: unsupervised learning, supervised learning, and unsupervised adaptive learning. The unsupervised learning algorithm collected data from the IMU during the calibration phase and derivated it to remove

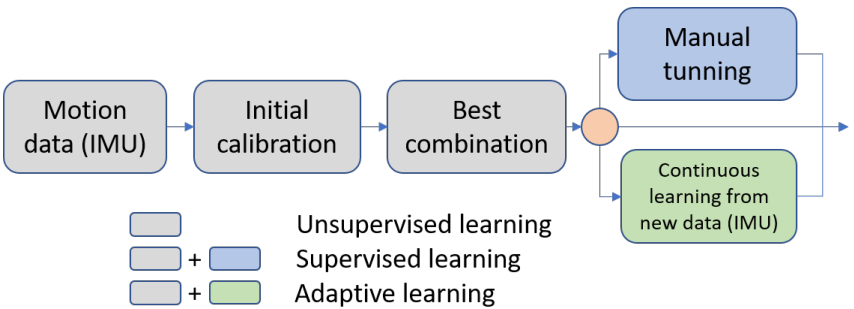

Figure 3. Learning processes block diagram.

the steady state component and to ignore initial angular positions. Thresholds were then calculated according to Eq. 1.

$$
T=\frac{\max \left(\frac{d X}{d t}\right)}{2}
$$

where vector $X$ features the raw sensor measurements and vector $T$ is the threshold vector. An overlaying moving window of 1 second was defined. Whenever its center value was greater than a threshold, a movement was detected and characterized as a 6-dimension point based on the Root Mean Square (RMS) of each of the 6 axis. A PCA is then applied to these points and the two first principal components are used to find two movement centroids based on the mean 2-dimensions coordinates of all points. After that the system calculates the centroids and points on all possible combination of two movements. It then proposes the best combination based on the highest score $s$, defined by Eq. 2 .

$$
s=\frac{d_{1,2}}{\sigma_{1}+\sigma_{2}}
$$

where $d_{1,2}$ is the distance between the two centroids, and $\sigma_{i}$ is the standard deviation of the distance of all points of movement $i$ to that movement centroid.

After calibration, the system could start classifying new movements associating the new PCA calculated 2dimensional point to the closest centroid, and activating the appropriate robotic hand command.

On the supervised learning algorithm the user performed the previously calibrated movements and watched the robotic hand reaction. We then observed the PCA graphical representations of these points in real time. If the new points seemed to appear far from the initially calibrated ones, we recalibrated the system with them, and without the old ones. It was also possible to arbitrarily choose each centroid location on the 2-dimensional space. With these tools we were able to manually tune the learning process until the users felt they had good control over the robotic hand.

On the adaptive learning algorithm, this process of improving the calibration is automated. After every new movement during the validation phase, the system is recalibrated using the new point, removing the oldest one.

Note that, differently from the supervised learning system, the other two are completely automatic. The three systems are summarized in Fig. 3. Users performed the experiments with the supervised learning algorithm. All data was recorded to later simulate the two other algorithms. 
Table I

MEAN PERFORMANCE RESULT ON THE RANDOM 10 MOVEMENTS SEQUENCE TEST. THE SUPERVISED LEARNING SYSTEM SCORES WERE OBTAINED FROM TESTS WITH THE SUBJECTS, WHILE THE OTHER TWO WERE SIMULATED.

\begin{tabular}{|c|c|c|c|}
\hline Subject & \multicolumn{3}{|c|}{ Performance [\%] } \\
\hline & Supervised & Unsupervised & Adaptive \\
\hline \hline p1 & 90 & 70 & 60 \\
\hline p2 & 100 & 100 & 100 \\
\hline p3 & 90 & 50 & 90 \\
\hline p4 & 70 & 70 & 70 \\
\hline p5 & 100 & 60 & 100 \\
\hline p6 & 70 & 70 & 80 \\
\hline p7 & 90 & 90 & 100 \\
\hline p8 & 100 & 100 & 100 \\
\hline Average & 89 & 76 & 88 \\
\hline
\end{tabular}

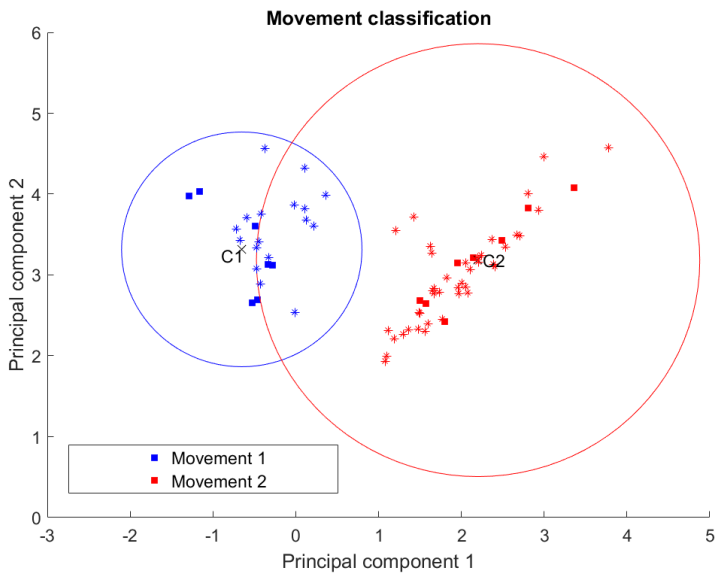

Figure 4. Supervised learning and classification for subject p8. The two axis represent the two principal components. Squares represent the movements used for learning and the asterisks the movements classified by the system. $\mathrm{C} 1$ and $\mathrm{C} 2$ are the centroids for movements 1 and 2, respectively. The circles, for visualization purposes only, represent three standard deviations of the euclidean distance of the squares to their centroids.

\section{RESULTS}

The average performance achieved experimentally with the supervised algorithm and through simulation with the other two is shown on Table I. Results were calculated as the percentage of correctly classified commands. Note how the unsupervised had inferior results $(76 \%)$ when compared to the supervised (89\%) and adaptive (88\%). Additionally, the adaptive learning algorithm would have the best performance were it not for patient $\mathrm{p} 1$.

After the initial calibration phase, it was possible to analyze its results on a graphic that represents the two main components of the PCA. Fig. 4 shows an example of one experiment.

The adaptive learning algorithm was simulated with points from the validation phase. Fig 5 shows the performance obtained along all steps of the learning process. Note how the performance seems to further improve after the seventh point.

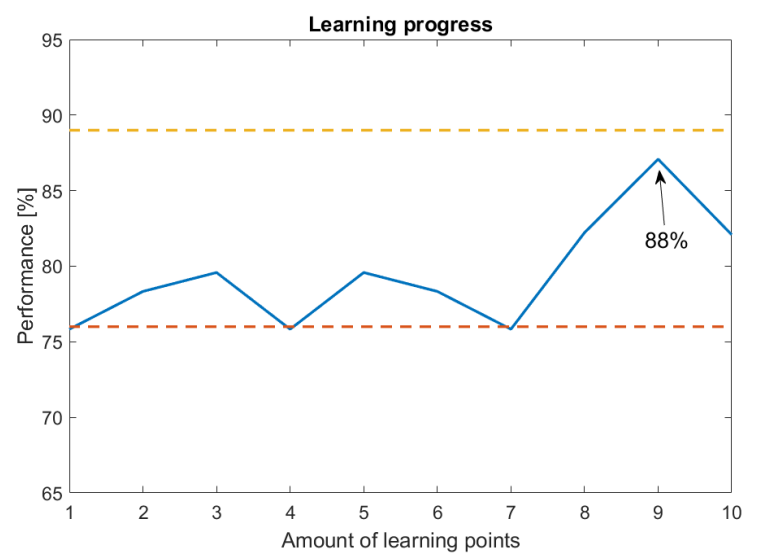

Figure 5. Adaptive learning algorithm process. The average best result was achieved when learning from 9 points. For comparison purposes, the red dashed line indicate the unsupervised learning system performance, and the yellow dashed line represent the supervised learning system performance.

\section{DISCUSSION}

We developed a system capable of classifying two shoulder movements performed by persons with tetraplegia with $89 \%$ accuracy. Even though the performance achieved by [9] $(96 \%)$ was greater, tests were done only on able-bodied subjects. Both [8] and [3] used multiple IMUs and EMG, with many more features than this work, to classify upper limb movement with LDA, the latter achieving results close to $80 \%$ on a 6 classes scenario. It is expected users with tetraplegia to have lower dexterity than healthy subjects, but our results indicate that, given more training for both users and system, better results are achievable. Furthermore, in comparison to the EMG approach, our system is capable of classifying multiple movements with a single IMU.

During the calibration phase, graphical visualizations as presented on Fig. 4 helped to assess the supervision process and manually tune the calibration. This, therefore, was a subjective process. Still, we were able to tune the system so the supervised learning algorithm presented the best results, while the unsupervised learning method presented the worst, highlighting the importance of the supervision on learning tasks with low informations level.

It was clear the difference in subject attitude and performance between the calibration (no feedback) and the validation (visual feedback from the robotic hand) phases. It was not unusual for them to change the movements then. When that happened, if no supervision was allowed, we would see results as the one shown on Fig. 6. That explains some of the bad performances, as subject p5's on the unsupervised algorithm. Nevertheless, these are also the cases when the adaptive learning performs best because it is capable of forgetting those initial trials and "focus" only on the recent ones. Note how p5's performance improves both on the supervised and adaptive algorithms. Were the adaptive algorithm working on real time, we expect its performance would improve in a similar manner as depicted on Fig. 5. Still, visual feedback representing a deeper functioning of 


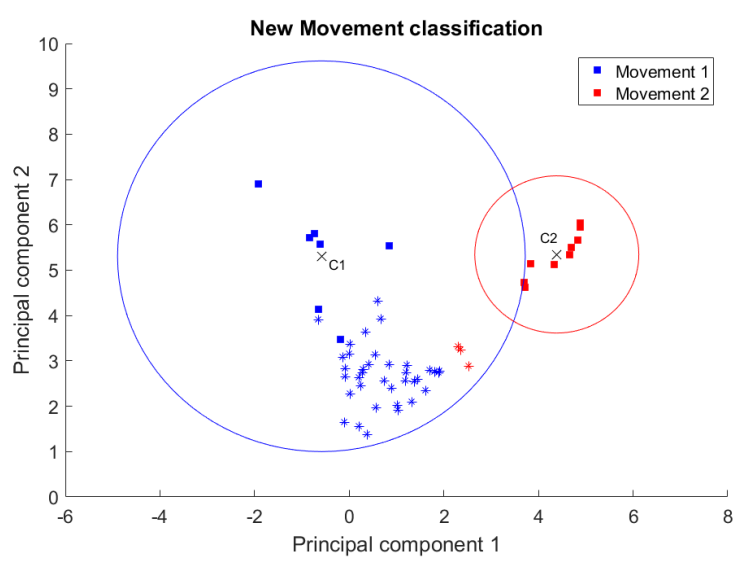

Figure 6. Unsupervised learning system test for subject p5. Note how almost all movements were classified as Movement 1 , and they were significantly different than those initially calibrated (square points).

the system and user's performance may be beneficial [10].

Observing Fig. 5, we can see the performance only improves after many new points are considered for learning. It possibly happens because the system still recalls the original, outdated points. As soon as the new points become a majority, their cumulative weight overcomes the old ones. It is also important to note that the adaptive learning system is fully automated, which can be an advantage on real world scenarios, when a user would possibly calibrate and operate it without specialized assistance. Since only the supervised learning system was tested by the subjects, and not the adaptive, such scenarios are yet to be evaluated.

Although no subject had cognitive impairments, some of them presented difficulties on operating the system. Most of them had never seen a robotic hand, and none was ever able to control one. In deed, novel technologies and control interfaces may initially be hard to use [10]. As a result, sometimes they lost focus on the task, and performed careless movements. That was particularly true for subject p6. Once again, in this case, we can see a slight improvement when using the adaptive learning system, highlighting the importance of the algorithm adaptability, either supervised or not.

In a larger clinical trial, our system should be adapted to control a more functional neuroprosthesis, e.g. FES on forearm to control grasping. The current finite state machine requires every posture to return to the rest position before performing a new one, which was useful for the algorithm evaluation, but is not practical. In addition, triggering the predefined movements works well for complex tasks as grasping, but lacks fine control like proportional force or hand opening range. This, too, could be addressed with residual shoulder movement [10], and could even be integrated in a larger finite state machine. Nevertheless, the simple setup of a single commercial IMU and flexible, quick learning algorithm provide easy adaptation and scalability.

\section{CONCLUSION}

Complex neuroprosthesis can be controlled by residual movement in users with tetraplegia. We developed a system capable of classifying different shoulder movements using a single wireless IMU with only accelerometer and gyroscope. We found that results close to $90 \%$ could be achieved either by a supervised system or by a unsupervised adaptive system with minimum user training. Regardless of the approach, the calibration process is simple and fast. To the extend of our knowledge, this is the first work to perform movement classification experiments where subjects with tetraplegia controlled a robotic hand. Future works include longer training and sessions with functional daily life activities.

\section{REFERENCES}

[1] Lotte N. S. Andreasen Struijk, Line Lindhardt Egsgaard, Romulus Lontis, Michael Gaihede, and Bo Bentsen. Wireless intraoral tongue control of an assistive robotic arm for individuals with tetraplegia. Journal of NeuroEngineering and Rehabilitation, 14(1):110, dec 2017.

[2] R. L. Hart, K. L. Kilgore, and P. H. Peckham. A comparison between control methods for implanted FES hand-grasp systems. IEEE Transactions on Rehabilitation Engineering, 6(2):208-218, 1998.

[3] Agamemnon Krasoulis, Iris Kyranou, Mustapha Suphi Erden, Kianoush Nazarpour, and Sethu Vijayakumar. Improved prosthetic hand control with concurrent use of myoelectric and inertial measurements. Journal of NeuroEngineering and Rehabilitation, 14(1):71, dec 2017.

[4] Joan Lobo-Prat, Peter N. Kooren, Arno Ha Stienen, Just L. Herder, Bart F.J.M. Koopman, and Peter H. Veltink. Non-invasive control interfaces for intention detection in active movement-assistive devices. Journal of NeuroEngineering and Rehabilitation, 11(1):1-22, 2014.

[5] Manelle Merad, Etienne de Montalivet, Amélie Touillet, Noël Martinet, Agnès Roby-Brami, and Nathanaël Jarrassé. Pre-clinical evaluation of a natural prosthetic elbow control strategy using residual limb motion and a model of healthy inter-joint coordinations. Annals of Physical and Rehabilitation Medicine, 60:e100, sep 2017.

[6] P.Hunter Peckham, Michael W. Keith, Kevin L. Kilgore, Julie H. Grill, Kathy S. Wuolle, Geoffrey B. Thrope, Peter Gorman, John Hobby, M.J. Mulcahey, Sara Carroll, Vincent R. Hentz, Allen Wiegner, and Implantable Neuroprosthesis Research Group. Efficacy of an implanted neuroprosthesis for restoring hand grasp in tetraplegia: A multicenter study. Archives of Physical Medicine and Rehabilitation, 82(10):13801388, oct 2001.

[7] Dejan Popovic and Thomas Sinkjaer. Control of Movement for the Physically Disabled. Springer London, London, 2000.

[8] Ashkan Radmand, Erik Scheme, and Kevin Englehart. On the Suitability of Integrating Accelerometry Data with Electromyography Signals for Resolving the Effect of Changes in Limb Position during Dynamic Limb Movement. Journal of Prosthetics and Orthotics, 26(4):185-193, oct 2014.

[9] Peter Sarcevic, Zoltan Kincses, and Szilveszter Pletl. Wireless Sensor Network based movement classification using wrist-mounted 9DOF sensor boards. In 2014 IEEE 15th International Symposium on Computational Intelligence and Informatics (CINTI), pages 85-90. IEEE, nov 2014.

[10] Elias B Thorp, Farnaz Abdollahi, David Chen, Ali Farshchiansadegh, Mei-Hua Lee, Jessica P Pedersen, Camilla Pierella, Elliot J Roth, Ismael Seanez Gonzalez, and Ferdinando A Mussa-Ivaldi. Upper Body-Based Power Wheelchair Control Interface for Individuals With Tetraplegia. IEEE transactions on neural systems and rehabilitation engineering, 24(2):249-60, feb 2016.

[11] Wafa Tigra, Benjamin Navarro, Andrea Cherubini, Xavier Gorron, Anthony Gelis, Charles Fattal, David Guiraud, and Christine Azevedo Coste. A novel EMG interface for individuals with tetraplegia to pilot robot hand grasping. IEEE Transactions on Neural Systems and Rehabilitation Engineering, 2016.

[12] B Wodlinger, J E Downey, E C Tyler-Kabara, A B Schwartz, M L Boninger, and $\mathrm{J} \mathrm{L}$ Collinger. Ten-dimensional anthropomorphic arm control in a human brain-machine interface: difficulties, solutions, and limitations. Journal of Neural Engineering, 12(1):016011, feb 2015. 